\title{
Consenso | Protocolo Brasileiro para Infecções Sexualmente Transmissíveis 2020: vigilância epidemiológica
}

doi: 10.1590/S1679-4974202100002.esp1

\author{
Brazilian Protocol for Sexually Transmitted Infections 2020: epidemiological surveillance \\ Protocolo Brasileño para Infecciones de Transmisión Sexual 2020: vigilancia epidemiológica
}

\author{
Carmen Silvia Bruniera Domingues ${ }^{1}$ - (D) orcid.org/0000-0003-2784-9592 \\ Leonor Henriette de Lannoy ${ }^{2}$ - (1) orcid.org/0000-0002-9520-8538 \\ Valeria Saraceni ${ }^{3}$ - (1) orcid.org/0000-0001-7360-6490 \\ Alessandro Ricardo Caruso da Cunha ${ }^{2}$ - (D) orcid.org/0000-0001-5441-1006 \\ Gerson Fernando Mendes Pereira² - (1) orcid.org/0000-0001-8886-1662 \\ 1Secretaria de Estado da Saúde de São Paulo, Centro de Referência e Treinamento de Doenças Sexualmente Transmissíveis e \\ Aids, São Paulo, SP, Brasil \\ ${ }^{2}$ Ministério da Saúde, Secretaria de Vigilância em Saúde, Brasília, DF, Brasil \\ ${ }^{3}$ Secretaria Municipal de Saúde do Rio de Janeiro, Rio de Janeiro, RJ, Brasil
}

\section{Resumo}

0 artigo sintetiza o capítulo sobre vigilância epidemiológica das infecções sexualmente transmissíveis (IST), que compõe 0 Protocolo Clínico e Diretrizes Terapêuticas (PDCT) para Atenção Integral às Pessoas com IST 2020, publicado pela Secretaria de Vigilância em Saúde, do Ministério da Saúde do Brasil. São apresentadas algumas reflexões sobre as novas definições de caso, para vigilância da sífilis adquirida, sífilis em gestantes e sífilis congênita. 0 PCDT-IST 2020 foi elaborado com base em evidências científicas e validado em discussões com especialistas. Também são abordados aspectos epidemiológicos e clínicos, bem como orientações para gestores no manejo programático e operacional desses agravos, e para profissionais de saúde na triagem, diagnóstico e tratamento das pessoas com IST e suas parcerias sexuais, além de estratégias de ação para a vigilância, prevenção e controle.

Palavras-chave: Vigilância em Saúde Pública; Sistemas de Informação em Saúde; Sífilis; Sífilis Congênita; Notificação de Doenças; Monitoramento Epidemiológico.

\section{Endereço para correspondência:}

Carmen Silvia Bruniera Domingues - Secretaria de Estado da Saúde de São Paulo, Centro de Referência e Treinamento de Doenças Sexualmente Transmissíveis e Aids, Programa Estadual de IST/Aids de São Paulo, Rua Santa Cruz, n 81, $1^{\circ}$ andar, Vila Mariana, São Paulo, SP, Brasil. CEP: 04121-000

E-mail: carmen@crt.saude.sp.gov.br 


\section{Apresentação}

0 artigo sintetiza o capítulo sobre vigilância epidemiológica das infecções sexualmente transmissíveis (IST), que compõe o Protocolo Clínico e Diretrizes Terapêuticas (PCDT) para Atenção Integral às Pessoas com IST, publicado pela Secretaria de Vigilância em Saúde, do Ministério da Saúde do Brasil. Para a elaboração do PCDT foram realizadas a seleção e a análise das evidências disponíveis na literatura, seguida de discussão, no formato de um painel de especialistas, para elaboração de recomendações. 0 PCDT foi aprovado pela Comissão Nacional de Incorporação de Tecnologias no Sistema Único de Saúde (Conitec) ${ }^{1}$, e atualizado pelo grupo de especialistas em IST do PCDT-IST $2020 .^{2}$

\section{As infecções sexualmente transmissiveis estão entre as doenças mais comuns em todo o mundo. Afetam a saúde e a vida das pessoas, impactam a saúde reprodutiva e infantil, contribuem para a infertilidade, complicações durante a gravidez e o parto.}

\section{Introdução}

As IST são causadas por vírus, bactérias ou outros microrganismos, transmitidas principalmente no contato sexual sem uso de preservativos. ${ }^{2}$ Consideradas um problema de saúde pública, estão entre as doenças mais comuns em todo o mundo. Elas afetam a saúde e a vida das pessoas, causam um impacto importante sobre a saúde reprodutiva e infantil, contribuem para a infertilidade, complicações durante a gravidez e o parto, e facilitam a transmissão sexual do vírus da imunodeficiência humana (buman immunodeficiency virus, HIV); e, extremo caso, podem levar à morte fetal. ${ }^{3}$

No Brasil, as bases para prevenção, diagnóstico e tratamento das IST estão bem estabelecidas, bem como seu modelo de vigilância epidemiológica, composto por notificação compulsória, serviços sentinela e estudos transversais em determinados grupos populacionais. ${ }^{4}$
As IST que fazem parte da lista nacional de notificação compulsória ${ }^{5,6}$ incluem os casos de síndrome da imunodeficiência adquirida (acquired immunodeficiency syndrome, aids), HIV, HIV em gestantes, hepatites virais, sífilis em gestantes, sífilis adquirida e síndrome do corrimento uretral masculino ${ }^{7}$ (Figura 1). 0s protocolos de HIV e hepatites são específicos e tratados separadamente. ${ }^{8-11} \mathrm{Em}$ todo o território nacional, a notificação compulsória da sífilis congênita, da sífilis em gestantes e da sífilis adquirida teve início em 1986, 2005 e 2010, respectivamente (Figura 1).

Os casos suspeitos ou confirmados constantes da lista nacional de notificação compulsória são notificados por meio do Sistema de Informação de Agravos de Notificação (Sinan) e seguem um fluxo ascendente, iniciado nas vigilâncias municipais, passando pelas estaduais, até a nacional. Em cada etapa desse processo, os diversos atores envolvidos têm a responsabilidade de coletar, analisar e divulgar as informações geradas pelo Sistema Nacional de Vigilância Epidemiológica, contribuindo para a melhoria da situação de saúde, subsidiando o planejamento e a adoção das medidas de controle necessárias ao conjunto ou grupos específicos da população brasileira, como também 0 monitoramento e avaliação das políticas, planos e programas de saúde. ${ }^{12}$

Este artigo teve como objetivo apresentar algumas reflexões sobre as novas definições de caso para a vigilância da sífilis adquirida, sífilis em gestantes e sífilis congênita, de acordo com o PCDT para Atenção Integral às Pessoas com IST $2020 .^{2}$

\section{Aspectos epidemiológicos}

Com base nos dados de prevalência de 2009 a 2016, a Organização Mundial da Saúde (OMS) estimou um total de 376,4 milhões de casos incidentes de IST, considerados curáveis. Desse total, 127,2 milhões ( $\left.\mathrm{IC}_{95 \%} 95,1 ; 165,9\right)$ eram casos estimados de clamídia, 86,9 milhões $\left(\mathrm{IC}_{95 \%}, 58,6 ; 123,4\right)$ de gonorreia, 156,0 milhões $\left(\mathrm{IC}_{95 \%} 103,4 ; 231,2\right)$ de tricomoníase e 6,3 milhões $\left(\mathrm{IC}_{95 \%} 5,5 ; 7,1\right)$ de sífilis. A prevalência global estimada de sífilis em homens e mulheres foi de $0,5 \%\left(\mathrm{IC}_{95 \%} 0,4 ; 0,6\right)$, com valores regionais variando de $0,1 \mathrm{a} 1,6 \%{ }^{13}$

No Brasil, a prevalência da sífilis pode ser estimada por meio de estudos transversais, 


\begin{tabular}{|c|c|c|}
\hline Agravo & Notificação instituída em: & Portaria \\
\hline Aids $^{\mathrm{a}}$ & 1986 & Portaria n 542, de 22 de dezembro de 1986 \\
\hline Sífilis congênita & 1986 & Portaria nº 542, de 22 de dezembro de 1986 \\
\hline Hepatite B & 1998 & Portaria n ${ }^{0} 4.052$, de 23 de dezembro de 1998 \\
\hline Hepatite C & 1999 & Portaria n ${ }^{0} 1.461$, de 22 de dezembro de 1999 \\
\hline 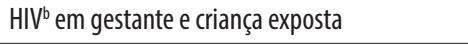 & 2000 & Portaria no 993, de 4 de setembro de 2000 \\
\hline Hepatites virais (inclusão das hepatites A, D e E) & 2003 & Portaria $\mathrm{n}^{0} 2.325$, de 8 de dezembro de 2003 \\
\hline Sífilis em gestantes & 2005 & Portaria no 33, de 14 de julho de 2005 \\
\hline Sífilis adquirida & 2010 & Portaria no 2.472, de 31 de agosto de 2010 \\
\hline HIV $^{b}$ & 2014 & Portaria no 1.271, de 6 de junho de 2014 \\
\hline
\end{tabular}

Notas: a) Aids: síndrome da imunodeficiência adquirida; b) HIV: vírus da imunodeficiência humana.

Figura 1 - Portarias que instituíram a notificação compulsória das infecções sexualmente transmissíveis e sífilis congênita, Brasil

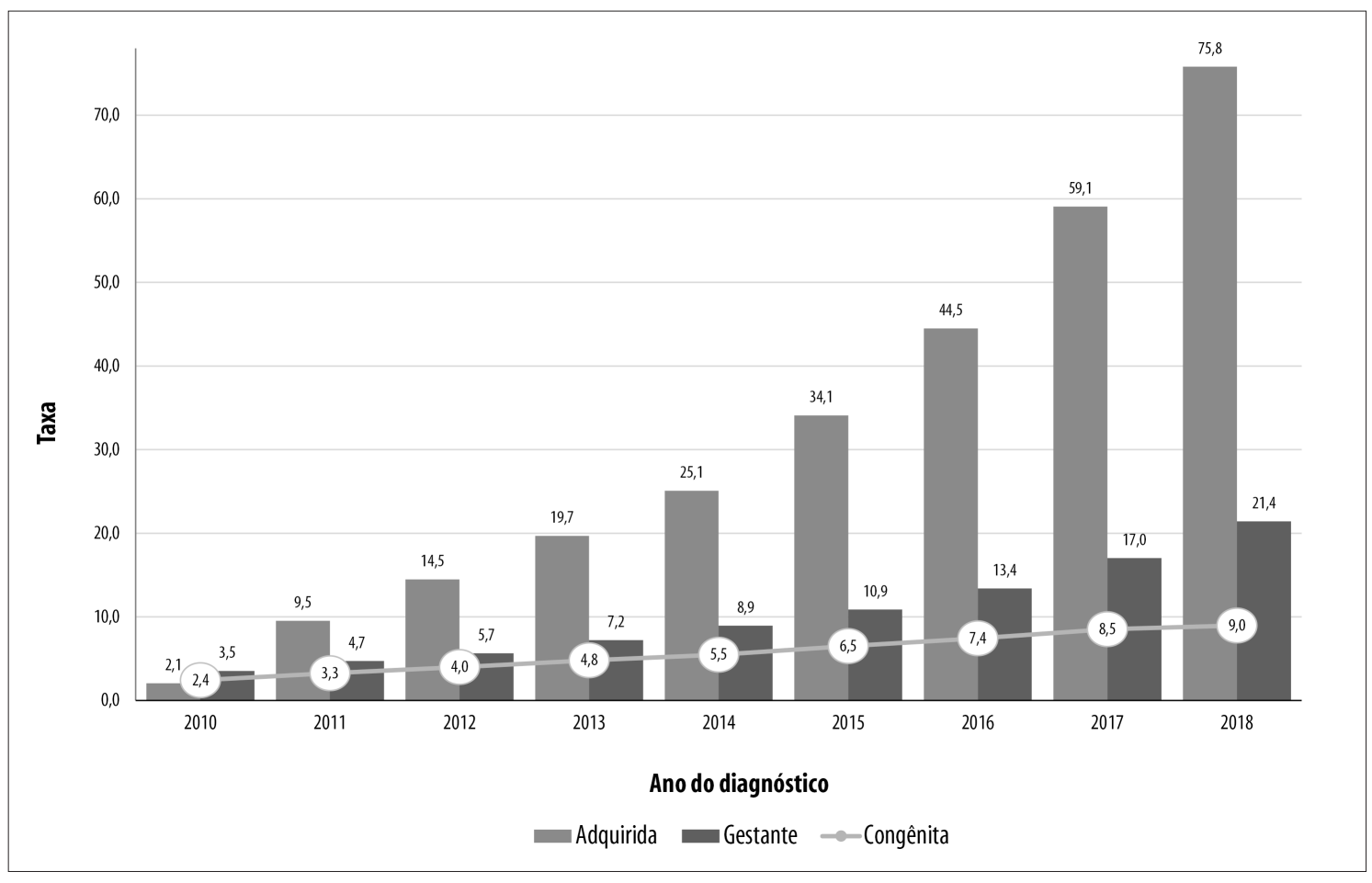

Fonte: Sistema de Informação de Agravos de Notificação.

Figura 2 - Taxa de deteç̧ão de sífilis adquirida (por 100 mil habitantes), taxa de deteç̧ão de sífilis em gestantes e taxa de incidência de sífilis congênita (por mil nascidos vivos) segundo ano de diagnóstico, Brasil, 2010-2018 
realizados em populações específicas. ${ }^{14-19} 0$ grupo de parturientes vinha sendo um dos alvos desses estudos, monitorado por apresentar prevalência semelhante à da população geral feminina. As estimativas nacionais variaram de $1,7 \%\left(\mathrm{IC}_{95 \%} 1,2 ; 2,2\right)$, no ano 2000, a 1,02\% (IC $\left.{ }_{95 \%} 0,84 ; 1,25\right)$ em 2011-2012. ${ }^{14-17}$ Entre jovens conscritos brasileiros, a prevalência de sífilis ativa foi estimada pela última vez em 2016, alcançando 0 valor de $1,1 \%\left(\mathrm{IC}_{95 \%} 0,85 ; 1,40\right) .{ }^{18} \mathrm{~A}$ estimativa de sífilis ativa em mulheres profissionais do sexo variou de 2,4\% ( $\left.\mathrm{IC}_{95 \%}, 1,7 ; 3,4\right)$ em 2009 a $8,5 \%$ $\left(\mathrm{IC}_{95 \%} 7,3 ; 9,7\right)$ em 2016. ${ }^{19}$

0 crescente número de casos tem indicado a persistência desses agravos, os quais, apesar de evitáveis, continuam a desafiar os sistemas de saúde. 0 país registrou 650.258 casos de sífilis adquirida entre $2010 \mathrm{e}$ 30/06/2019, 324.321 de sífilis em gestantes entre 2005 e 30/06/2019, e 214.891 de sífilis congênita entre 1998 e 30/06/2019. ${ }^{20}$ No período de 2014 a 2018 (Figura 2), a taxa de detecção de sífilis adquirida elevou-se em três vezes (passou de 25,1 para 75,8 casos por 100 mil habitantes); a de sífilis em gestantes, em 2,4 vezes (passou de 8,9 para 21,4 casos por mil nascidos vivos); e a taxa de incidência de sífilis congênita em 1,6 vez (passou de 5,5 para 9,0 casos por mil nascidos vivos). Em 2018, a relação das taxas de detecção de sífilis em gestantes e incidência de sífilis congênita foi de 2,4 gestantes com sífilis para uma criança com sífilis congênita, sendo que em 14 Unidades da Federação (UFs), essa relação encontra-se abaixo do patamar nacional (Figura 3). A incidência da sífilis congênita ainda apresenta valores muito acima dos estabelecidos pela OMS para eliminação desse agravo. ${ }^{21}$

Não obstante a elevada detecção de casos, é sabido que a sífilis é um agravo subnotificado, ${ }^{22,23} \mathrm{com}$ implicações para a resposta às IST no país, dado o número total de ocorrências e aspectos relacionados a comportamento e vulnerabilidade. A subestimação de casos pode comprometer o planejamento do sistema de saúde, no que concerne ao fornecimento contínuo

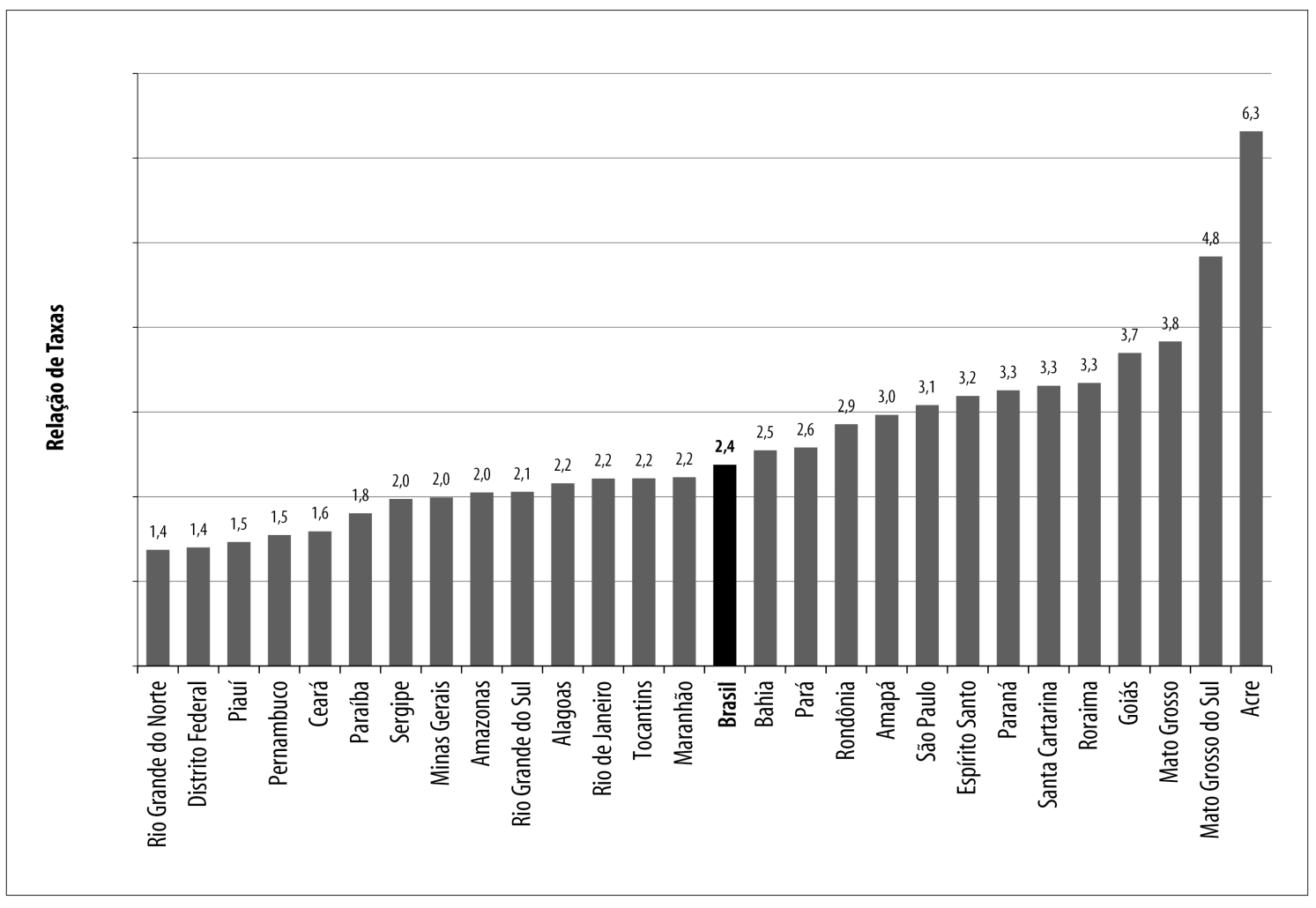

Fonte: Sistema de Informação de Agravos de Notificação.

Figura 3 - Relação da taxa de deteç̧ão de sífilis em gestantes e taxa de incidência de sífilis congênita segundo Unidades da Federação, Brasil, 2018 
de insumos e implementação de ações prioritárias, principalmente aquelas dirigidas às populações com maior vulnerabilidade. ${ }^{2}$

Em 2017, para se alinhar às recomendações da Organização Pan-Americana da Saúde (OPAS), o Ministério da Saúde atualizou a definição de caso de sífilis adquirida, sífilis em gestantes e sífilis congênita, por meio da Nota Informativa $\mathrm{n}^{0} 2-\mathrm{SEI} / 2017$ - DIAHV/SVS/MS. ${ }^{24}$ Entretanto, o formato eletrônico do Sinan não foi adaptado às modificações promovidas nas fichas, trazendo algumas dificuldades para o alinhamento dos critérios no nível dos serviços de

\section{Critérios para definição de caso de sífilis congênita}

\section{Situação 1}

Todo recém-nascido, natimorto ou aborto de mulher com sífilisa não tratada ou tratada de forma não adequada. ${ }^{\mathrm{b}, \mathrm{c}}$

\section{Situação $2^{\mathrm{d}}$}

Toda criança com menos de 13 anos de idade com pelo menos uma das seguintes situações:

- Manifestação clínica, alteração liquórica ou radiológica de sífilis congênita e teste não treponêmico reagente.

- Títulos de teste não treponêmicos do lactente maiores que os da mãe, em pelo menos duas diluições de amostras de sangue periférico, coletadas simultaneamente no momento do parto.

- Títulos de testes não treponêmicos ascendentes em pelo menos duas diluições no seguimento da criança exposta. ${ }^{\text {e }}$

- Títulos de testes não treponêmicos ainda reagentes após 6 meses de idade, exceto em situação de seguimento terapêutico.

- Testes treponêmicos reagentes após 18 meses de idade, sem diagnóstico prévio de sífilis congênita.

\section{Situação 3}

Evidência microbiológica ${ }^{f}$ de infecção pelo Treponema pallidum em amostra de secreção nasal ou lesão cutânea, biópsia ou necropsia de criança, aborto ou natimorto.

a) Ver definição de sífilis em gestantes.

b) Tratamento adequado: tratamento completo para estágio clínico de sífilis com benzilpenicilina benzatina, iniciado até 30 dias antes do parto.

Gestantes que não se enquadrarem nesses critérios serão consideradas como tratadas de forma não adequada.

c)Para fins de definição de caso de sífilis congênita, não se considera o tratamento da parceria sexual da mãe.

d)Nessa situação, deve ser sempre afastada a possibilidade de sífilis adquirida em situação de violência sexual.

e) Seguimento da criança exposta: 1, 3, 6, 12 e 18 meses de idade.

f)Detecção do Treponema pallidum por meio de exames diretos por microscopia (de campo escuro ou com material corado).

Nota: a parceria sexual da gestante com sífilis NÃO faz parte da definição de caso de sífilis congênita, em relação ao tratamento materno, se adequado ou não adequado. Todavia, o risco de reinfecção por sífilis deve ser acompanhado em todo o período do atendimento pré-natal. Assim, recomenda-se que todas as parcerias sexuais de mulheres grávidas com infecções sexualmente transmissíveis sejam avaliadas e tratadas, para evitar reinfecções nas gestantes que foram tratadas adequadamente, especialmente para a sífilis.

\section{Critérios para definição de caso de sífilis em gestantes}

\section{Situação 1}

Mulher assintomática para sífilis que, durante o pré-natal, parto ou puerpério, apresente pelo menos um teste reagente - treponêmico ou não treponêmico com qualquer titulação - e que não tenha registro de tratamento prévio.

\section{Situação 2}

Mulher sintomáticaa para sífilis que, durante o pré-natal, parto ou puerpério, apresente pelo menos um teste reagente - treponêmico ou não treponêmico - com qualquer titulação

\section{Situação 3}

Mulher que, durante o pré-natal, parto ou puerpério, apresente teste não treponêmico reagente com qualquer titulação e teste treponêmico reagente, independentemente de sintomatologia de sífilis e sem registro de tratamento prévio.

a)Para mais informações sobre sífilis, consultar o Guia de Vigilância em Saúde e e os capítulos 5 e 6 do Protocolo Clínico e Diretrizes Terapêuticas para Atenção Integral às Pessoas com Infecções Sexualmente Transmissíveis 2020.2

Nota: todos os casos de mulheres diagnosticadas com sífilis durante 0 atendimento pré-natal, parto ou puerpério devem ser notificados na ficha de sífilis em gestantes. Casos confirmados de "cicatriz sorológica" não devem ser notificados. Considera-se "cicatriz sorológica" um tratamento anterior para sífilis com documentação da queda da titulação em pelo menos duas diluições (ex.: uma titulação de 1:16 antes do tratamento que se torna menor ou igual a 1:4 após o tratamento). 


\begin{tabular}{l} 
Continução \\
\begin{tabular}{|l|}
\hline Critérios para definição de caso de sífilis adquirida \\
\hline Situação 1 \\
Indivíduo assintomático, com teste não treponêmico reagente com qualquer titulação e teste treponêmico reagente, sem registro de tratamento prévio. \\
\hline Situação 2 \\
Indivíduo sintomáticoa para sífilis, com pelo menos um teste reagente - treponêmico ou não treponêmico - com qualquer titulação. \\
\hline a)Para mais informações sobre sífilis, consultar o Guia de Vigilância em Saúde ${ }^{8}$ e os capítulos 5 e 6 do Protocolo Clínico e Diretrizes Terapêuticas para \\
Atenção Integral às Pessoas com Infecções Sexualmente Transmissíveis $2020^{2}$ \\
Nota: casos de mulheres diagnosticadas com sífilis durante o atendimento pré-natal, parto ou puerpério devem ser notificados na ficha de sífilis em \\
gestantes. Casos confirmados de "cicatriz sorológica" não devem ser notificados. Considera-se "cicatriz sorológica" um tratamento anterior para sífilis com \\
documentação da queda da titulação em pelo menos duas diluições (ex.: uma titulação de 1:16 antes do tratamento que se torna menor ou igual a 1:4 \\
após o tratamento).
\end{tabular} \\
\hline
\end{tabular}

Figura 4 - Critérios para definição de caso de sífilis congênita, sífilis em gestantes e sífilis adquirida, Brasil

saúde. A definição de caso é fundamental, deve ser clara e objetiva, pois bem compreendida, estabelece uma padronização que permite a um conjunto de critérios decidir se uma pessoa apresenta ou não determinada doença ou agravo, tornando os casos comparáveis na entrada do sistema de vigilância. ${ }^{25,26}$

A seguir, são apresentadas as definições de caso de sífilis adquirida, sífilis em gestantes e sífilis congênita (Figura 4).

\section{Sífilis adquirida}

Para fins de vigilância epidemiológica, foram estabelecidos dois critérios definidores de caso, denominados de Situação 1 e Situação 2, que permitem notificar a sífilis adquirida. A definição de caso é abrangente, possibilita a inclusão de indivíduos com sífilis em qualquer fase clínica da doença, especialmente quando classificados pelo primeiro critério ou Situação 1 (Figura 4).

A sífilis adquirida conta com uma ficha de notificação e investigação que pode ser utilizada pelas vigilâncias epidemiológicas municipais e estaduais. No Sinan, são inseridos apenas dados referentes à notificação do caso, ou seja, dados de identificação e demográficos da pessoa acometida. Espera-se que as variáveis correspondentes ao componente de investigação epidemiológica, como dados clínicos e laboratoriais, comportamento e vulnerabilidade, também sejam incluídas no sistema, para melhor compreensão do agravo.

É importante a disponibilização de informações sobre a fase clínica da doença, tipo de teste diagnóstico (teste treponêmico e/ou não treponêmico), tratamento realizado (benzilpenicilina benzatina ou outro medicamento) e outros elementos que possam contribuir para a análise epidemiológica, como também para a verificação do cumprimento da definição de caso estabelecida no país. Além disso, é fundamental a diferenciação entre duplicidades de caso e reinfecções - estas, quando o mesmo indivíduo foi notificado repetidas vezes.

Atualmente, no Brasil, o perfil da sífilis adquirida está vinculado à capacidade de detecção e notificação de casos pelos serviços de saúde, e às características demográficas das pessoas acometidas - sexo, idade, raça/ cor da pele, escolaridade, residência, outras. 0s dados podem se referir a casos prevalentes ou incidentes, haja vista não ser possível definir a fase clínica da doença. Seria importante identificar casos incidentes, classificados como sífilis recente, na forma clínica primária, secundária e latente recente (até um ano da infecção), período considerado de maior transmissibilidade do agente infeccioso, Treponema pallidum. ${ }^{2}$

\section{Sífilis em gestantes}

A nova definição de caso de sífilis em gestantes, caracterizada por três situações, tornou-se mais sensível e abrangente ao incluir as mulheres diagnosticadas no momento do parto ou no puerpério, contribuindo para ampliar a deteç̧ão da doença no período gravídico-puerperal (Figura 4). No entanto, as notificações de parturientes e puérperas diagnosticadas com essa enfermidade devem ser realizadas utilizando-se a ficha de notificação de sífilis em gestante, cujas variáveis de 


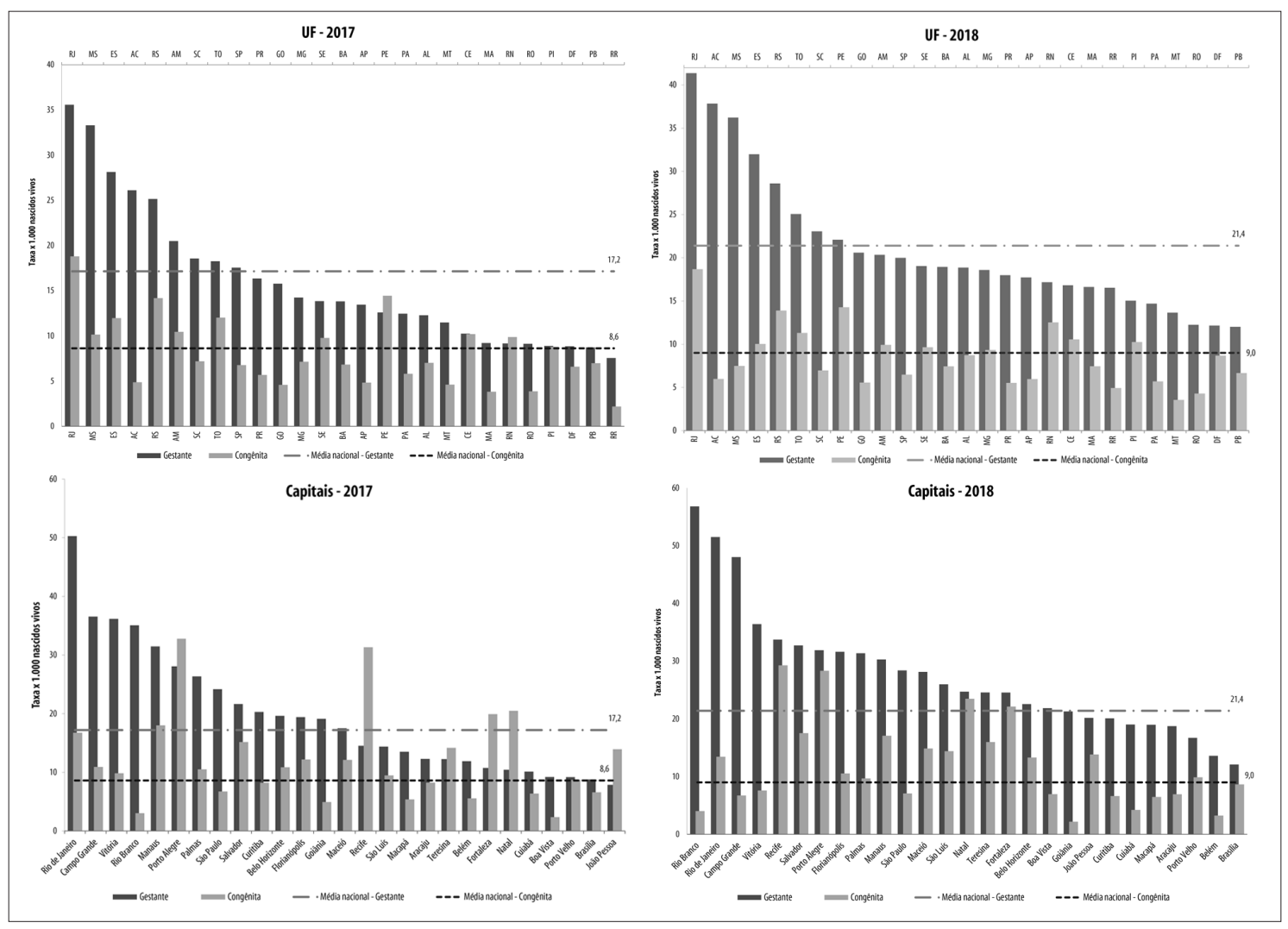

Nota: 2017 - Casos notificados de gestante com sífilis durante 0 atendimento pré-natal; 2018 - Casos notificados no pré-natal, parto ou puerpério. Fonte: Sistema de Informação de Agravos de Notificação.

\section{Figura 5 - Taxa de deteç̧ão de sífilis em gestantes e taxa de incidência de sífilis congênita (por mil nascidos vivos) segundo Unidade da Federação (UF) e capitais, Brasil, 2017-2018}

investigação estão vinculadas, em sua maioria, aos dados obtidos durante 0 atendimento pré-natal.

0 aumento das notificações de gestantes com sífilis, especialmente quando a detecção é feita no momento do parto ou no puerpério, pode não se refletir em redução da sífilis congênita, devido a perdas de oportunidades no tratamento materno. Em 2017, quando a notificação da gestante era realizada exclusivamente no pré-natal, foi possível observar taxas de incidência de sífilis congênita superiores às taxas de detecção de sífilis em gestantes, em algumas UF e capitais brasileiras. ${ }^{20}$ A partir de 2018, com a inserção de casos diagnosticados no parto ou puerpério, a taxa de detecção de sífilis em gestantes apresentou crescimento, embora sem grande impacto na redução da sífilis congênita (Figura 5).
$\mathrm{Na}$ classificação de caso pela Situação 1, existe a possibilidade de inclusão indevida de gestantes com resultados de testes discordantes, principalmente quando o teste não treponêmico é reagente e o teste treponêmico é não reagente. Muitas vezes, profissionais que realizam a notificação não se lembram de levar em consideração a possibilidade de resultado falso positivo do teste não treponêmico e por conseguinte, a necessidade de realizar outro teste treponêmico, com metodologia diferente. ${ }^{2}$ Todavia, essa definição é bastante sensível e permite a inclusão de casos quando os serviços não dispõem os dois tipos de teste disponíveis.

Na Situação 3, apesar de a nota de rodapé ressaltar que casos confirmados de "cicatriz sorológica" não devem ser informados ao sistema de vigilância, ainda é possível ocorrer notificações indevidas ou subnotificações, a depender do entendimento dos profissionais. 
0 conceito de "cicatriz sorológica" encontra-se descrito no PCDT-IST 2020, de modo a melhor informar sobre 0 significado de 'tratamento anterior para a sífilis.'

Uma nova revisão nos critérios de definição de caso de sífilis em gestantes, com o cuidado de não perder em sensibilidade, poderia contribuir para a qualidade dos casos notificados. Possivelmente, uma definição de caso com apenas dois critérios, portanto mais simples, seria de fácil entendimento para as vigilâncias epidemiológicas.

\section{Sífilis congênita}

Desde que adquiriu o status de notificação compulsória em 1986, a sífilis congênita tem sua definição de caso revisada periodicamente. Sua última atualização, publicada no final de 2017, estabeleceu três situações para a definição de caso (Figura 4). Uma das principais mudanças foi a exclusão do tratamento do parceiro sexual na descrição de tratamento materno adequado, contribuindo para que a definição de caso brasileira se tornasse mais específica e alinhada aos conceitos internacionais.

Em suas versões anteriores, os critérios de caso buscavam acentuar a sensibilidade, de modo a captar 0 maior número possível de ocorrências. Eles foram importantes, especialmente no momento quando o país ainda apresentava elevada subnotificação de casos e invisibilidade da sífilis congênita. ${ }^{27}$ É provável que a retirada do parceiro sexual materno, atuando de forma indireta na definição de caso de sífilis congênita, tenha produzido impacto na desaceleração da taxa de incidência da infecção. Entre 2016 e 2017, observou-se elevação dessa taxa em $15 \%$, de 7,4 para 8,5 casos por 1.000 nascidos vivos, respectivamente (Figura 2), ao passo que, entre 2017 e 2018, na vigência da nova definição de caso, o incremento na taxa de incidência foi de $6 \%$, de 8,5 para 9,0 casos por 1.000 nascidos vivos. ${ }^{20}$

A maior parte dos casos de sífilis congênita é classificada pela Situação 1, e a menor pela Situação 3. A realização de necropsia de criança, aborto ou natimorto, assim como a coleta de material de lesão cutânea ou de secreção nasal para detecção do Treponema pallidum em exames diretos, por microscopia, são diagnósticos limitados nos serviços hospitalares ou ambulatoriais. ${ }^{20}$

Em relação à Situação 2, observa-se que os profissionais de vigilância apresentam maior dificuldade para classificar os casos nos seguintes componentes: (i) títulos de testes não treponêmicos ascendentes em pelo menos duas diluições no seguimento da criança exposta; (ii) títulos de testes não treponêmicos ainda reagentes após seis meses de idade, exceto em situação de seguimento terapêutico; e (iii) testes treponêmicos reagentes após 18 meses de idade, sem diagnóstico prévio de sífilis congênita. Estes critérios são utilizados para definir caso de sífilis congênita na situação em que a criança for considerada como exposta a sífilis na maternidade e, durante o seguimento ambulatorial, apresentar alterações nos testes sorológicos. Eles também são utilizados para crianças maiores de 18 meses de idade, sem diagnóstico prévio de sífilis congênita ou de exposição à sífilis materna. Cumpre lembrar: deve ser afastada a possibilidade de sífilis adquirida por abuso sexual. ${ }^{2}$

A capacitação de profissionais da rede de Atenção Primária à Saúde é de suma importância na condução dos casos de sífilis congênita, assim como na vinculação das crianças expostas nos serviços ambulatoriais, procedimentos cuja atualização e aprimoramento são fundamentais para evitar perdas de oportunidades no diagnóstico, tratamento e subnotificação de casos de sífilis congênita detectados após alta da maternidade. .8,29 $^{28}$

É preciso envidar esforços para atingir a meta de eliminação de sífilis congênita proposta pela OPAS/OMS - 0,5 caso por mil nascidos vivos (incluídos os natimortos),$-{ }^{21}$ pois, apesar da mudança na definição de caso, tornando-a mais específica, o número de ocorrências mantém-se ascendente, ${ }^{20}$ contribuindo para a elevação da taxa de incidência a valores muito acima do esperado para sua eliminação (Figura 2).

\section{Considerações sobre vigilância, prevenção e controle das IST}

Refletindo sobre a vigilância de IST, nota-se que, em 2020, ainda não existem dados oficiais produzidos de forma sistemática, sobre gonorreia, clamídia, tricomoníase ou herpes genital no Brasil, uma vez que essas IST não fazem parte da lista nacional de notificação compulsória. ${ }^{6}$ Entretanto, é possível estimar sua prevalência com 0 desenvolvimento de estudos sobre determinadas populações, assistidas em serviços específicos.

0 Ministério da Saúde instituiu dois grandes projetos para as IST: 'Resposta Rápida à Sífilis', com a 
finalidade de reduzir a ocorrência de sífilis adquirida e em gestantes, e eliminar a sífilis congênita, ${ }^{30}$ e 'Infecções de transmissão sexual: Vigilância no Brasil da Etiologia das Uretrites e das Úlceras Genitais e Análise da Resistência aos Antimicrobianos', nomeado projeto SenGono, com o objetivo de monitorar a resistência antimicrobiana de cepas de Neisseria gonorrhoeae circulantes no país, identificar os principais agentes causadores de corrimento uretral e de úlceras genitais, utilizando-se de testes moleculares em serviços específicos. ${ }^{30,31}$

Importantes fontes de informações, além do Sinan, podem ser acessadas de modo rotineiro, pelas vigilâncias epidemiológicas municipais, estaduais e federais, para a obtenção de informações complementares sobre IST, a exemplo do Sistema de Informações sobre Mortalidade (SIM), Sistema de Informações sobre Nascidos Vivos (Sinasc), Sistema de Informações Hospitalares (SIH) e Sistema de Informações Ambulatoriais (SIA), e os sistemas de realização de exames complementares. ${ }^{32}$

A prevenção das IST permanece como desafio. A falta de conhecimento ou de percepção da situação da sífilis no país e seus determinantes sociais, as fragilidades do sistema de saúde, especialmente no acesso e na qualidade do atendimento pré-natal, são condições que contribuem para a persistência da sífilis congênita. ${ }^{33,34}$ É importante a manutenção do compromisso político com a saúde pública em todos os níveis de gestão, priorizando a prevenção, o diagnóstico precoce e o tratamento oportuno da sífilis, a estratégia mais efetiva para atingir a eliminação da sífilis congênita no Brasil..$^{35}$

Os sistemas de informações em saúde, especialmente o Sinan, são importantes ferramentas para as ações de vigilância. ${ }^{36}$ É fundamental a atualização e 0 alinhamento entre as revisões de definição de caso, a ficha de notificação e investigação epidemiológica, além do sistema de informações destinado a receber e preservar a base desses dados. A qualidade da informação será aprimorada à medida que os dados forem sistematicamente coletados, inseridos e processados nos sistemas, em conformidade com os critérios estabelecidos para as definições de caso.

As definições de caso de sífilis adquirida, sífilis em gestantes e sífilis congênita, pautadas em critérios suficientemente sensíveis e mais específicos, possibilitam a comparação do perfil epidemiológico brasileiro com o de outros países. Outrossim, produzem informações para a vigilância monitorar o comportamento e tendências da doença, com a finalidade de recomendar medidas de prevenção e controle, além de intensificar ações na atenção pré-natal, visando interromper a cadeia de transmissão vertical da sífilis.

\section{Contribuição dos autores}

Domingues CSB, de Lannoy LH, Saraceni V. Cunha ARC e Pereira GFM redigiram o manuscrito, aprovaram a versão final e são responsáveis por todos seus aspectos, incluindo a garantia de sua precisão e integridade.

\section{Agradecimento}

0s autores agradecem a contribuição substancial, para a realização deste trabalho, dos membros do grupo técnico de especialistas responsáveis pela elaboração do PCDT para Atenção Integral às Pessoas com IST 2020.

\section{Referências}

1. Brasil. Ministério da Saúde. Portaria MS/SCTIE $\mathrm{n}^{0}$ 42, de 05 de outubro de 2018. Torna pública a decisão de aprovar o Protocolo Clínico e Diretrizes Terapêuticas para Atenção Integral às Pessoas com Infecções Sexualmente Transmissíveis (IST), no âmbito do Sistema Único de Saúde - SUS [Internet]. Diário Oficial da União, Brasília (DF), 2018 out 08 [citado 2020 jul 31]; Seção 1:88. Disponível em: http://conitec.gov.br/images/Relatorios/Portaria/2018/Portaria_SCTIE_N42_05_10_2018.pdf
2. Ministério da Saúde (BR). Secretaria de Vigilância em Saúde. Departamento de Doenças de Condições Crônicas e Infecções Sexualmente Transmissíveis. Protocolo clínico e diretrizes terapêuticas para atenção integral às pessoas com infecções sexualmente transmissíveis (IST) [Internet]. Brasília: Ministério da Saúde; 2020 [citado 2020 ago 31]. Disponível em: http://www.aids.gov.br/pt-br/ pub/2015/protocolo-clinico-e-diretrizes-terapeuticas-para-atencao-integral-pessoas-com-infeccoes 
3. World Health Organization - WHO. Global health sector strategy on sexually transmitted infections 2016-2021: towards ending STIs [Internet]. Geneva: World Health Organization; 2016 [cited 2020 Jun 08]. 64p. Available from: https:/apps. who.int/iris/bitstream/handle/10665/250253/ WHO-RHR-16.09-spa.pdf;jsessionid=50EC035F2937217ED7E54D126CDF3784? sequence =1

4. Pereira GFM, Pimenta MC, Giozza SP, Caruso AR, Bastos FI, Guimarães MDC. HIV/aids, hepatites virais e outras IST no Brasil: tendências epidemiológicas. Rev Bras Epidemiol [internet]. 2019 set [citado 2020 jun 08];22(Suppl 1):e190001.supl1. Disponível em: https://doi.org/10.1590/1980-549720190001.supl.1

5. Brasil. Ministério da Saúde. Portaria MS/GM n ${ }^{0} 1.984$, de 12 de setembro de 2014. Define a lista nacional de doenças e agravos de notificação compulsória, na forma do Anexo, a serem monitorados por meio da estratégia de vigilância em unidades sentinelas e suas diretrizes [Internet]. Diário Oficial da União, Brasília (DF), 2014 set 15 [citado 2020 maio 31]; Seção 1:59. Disponível em: http://bvsms.saude.gov.br/ bvs/saudelegis/gm/2014/prt1984_12_09_2014.html

6. Brasil. Ministério da Saúde. Portaria de Consolidação MS/GM n ${ }^{0}$ 4, de 28 de setembro de 2017. Consolidação das normas sobre os sistemas e os subsistemas do Sistema Único de Saúde [Internet]. Diário Oficial da União, Brasília (DF), 2017 out 03 [citado 2020 jul 31];Suplemento:288. Disponível em: http://bvsms.saude.gov.br/ bvs/saudelegis/gm/2017/prc0004_03_10_2017.html

7. Brasil. Ministério da Saúde. Portaria MS/GM n ${ }^{0}$ 1.553, de 17 de junho de 2020. Altera a Portaria de Consolidação $n^{0}$ 5/GM/MS, de 28 de setembro de 2017, para instituir a Vigilância Sentinela da Síndrome do Corrimento Uretral Masculino (VSCUM) [Internet]. Diário Oficial da União, Brasília (DF), 2020 jun 18 [citado 2020 jun 29]; Seção 1:61. Disponível em: https:/www.in.gov.br/en/web/dou/-/ portaria-n-1.553-de-17-de-junho-de-2020-262147771

8. Ministério da Saúde (BR). Secretaria de Vigilância em Saúde. Coordenação Geral de Desenvolvimento da Epidemiologia em Serviços. Guia de vigilância em saúde: volume único [internet]. 3. ed. Brasília: Ministério da Saúde; 2019 [citado 2020 jul 31]. 740 p. Disponível em: http://bvsms.saude.gov.br/ bvs/publicacoes/guia_vigilancia_saude_3ed.pdf

9. Ministério da Saúde (BR). Secretaria de Vigilância em Saúde. Departamento de Vigilância, Prevenção e Controle das Infecções Sexualmente Transmissíveis, do HIV/Aids e das Hepatites Virais. Protocolo clínico e diretrizes terapêuticas para manejo da infecção pelo HIV em adultos [Internet]. Brasília: Ministério da Saúde; 2018. Disponível em: http:/www.aids.gov.br/ pt-br/pub/2013/protocolo-clinico-e-diretrizes-terapeuticas-para-manejo-da-infeccao-pelo-hiv-em-adultos

10. Ministério da Saúde (BR). Secretaria de Vigilância em Saúde. Departamento de Vigilância, Prevenção e Controle das Infecções Sexualmente Transmissíveis, do HIV/Aids e das Hepatites Virais. Protocolo clínico e diretrizes terapêuticas para hepatite B e coinfecções [Internet]. Brasília: Ministério da Saúde; 2016 [citado 2020 ago 31]. Disponível em: http://www. aids.gov.br/pt-br/pub/2016/protocolo-clinico-e-diretrizes-terapeuticas-para-hepatite-b-e-coinfeccoes

11. Ministério da Saúde (BR). Secretaria de Vigilância em Saúde. Departamento de Vigilância, Prevenção e Controle das Infecções Sexualmente Transmissíveis, do HIV/Aids e das Hepatites Virais. Protocolo clínico e diretrizes terapêuticas para hepatite $\mathrm{C}$ e coinfecções [Internet]. Brasília: Ministério da Saúde; 2017 [citado 2020 ago 31]. Disponível em: http://www. aids.gov.br/pt-br/pub/2017/protocolo-clinico-e-diretrizes-terapeuticas-para-hepatite-c-e-coinfeccoes

12. Brasil. Ministério da Saúde. Instrução Normativa $\mathrm{n}^{0} 2$, de 22 de novembro de 2005. Regulamenta as atividades da vigilância epidemiológica com relação à coleta, fluxo e a periodicidade de envio de dados da notificação compulsória de doenças por meio do Sistema de Informação de Agravos de Notificação - SINAN [Internet]. Diário Oficial da União, Brasília (DF), 2005 nov 22 [citado 2020 jun 10]. Disponível em: http://portalsinan.saude.gov.br/ images/documentos/Legislacoes/IN_2_2005.pdf

13. Rowley J, Hoorn SV, Korenromp E, Low N, Unemo M, Abu-Raddad LJ, et al.Chlamydia, gonorrhoea, trichomoniasis and syphilis: global prevalence and incidence estimates, 2016. Bull World Health Organ [Internet]. 2019 Aug [cited 2020 Jun 08];97(8):548-62. Available from: https://dx.doi.org/10.2471\%2FBLT.18.228486

14. Rodrigues CS, Guimarães MDG, Grupo Nacional de Estudo sobre Sífilis Congênita. Positividade para sífilis em puérperas: ainda um desafio para o Brasil. Rev Panam Salud Publica [internet]. 2004 [citado 2020 jun 09];16(3):168-75. Disponível em: https:// scielosp.org/pdf/rpsp/2004.v16n3/168-175/pt

15. Szwarcwald CL, Barbosa Jr A, Miranda AE, Paz LC. Resultados do estudo sentinela-parturiente, 2006: desafios para o controle da sífilis congênita no Brasil. 
DST - J Bras Doenças Sex Transm [Internet]. 2007 nov [citado 2020 jun 09];19(3-4):128-33. Disponível em: http://www.dst.uff.br/revista19-3-2007/3.pdf

16. Domingues RM, Szwarcwald CL, Souza Junior PR, Leal MC. Prevalence of syphilis in pregnancy and prenatal syphilis testing in Brazil: birth in Brazil study. Rev Saúde Pública [internet]. 2014 out [citado 2020 jun 09];48(5):766-74. Disponível em: https:// doi.org/10.1590/S0034-8910.2014048005114

17. Cunha ARC, Merchan-Hamann E. Sífilis em parturientes no Brasil: prevalência e fatores associados, $2010 \mathrm{a}$ 2011. Rev Panam Salud Publica [internet]. 2015 [citado 2020 jun 09];38(6):479-86. Disponível em: https:// www.scielosp.org/article/rpsp/2015.v38n6/479-486/

18. Freitas FLS. Sífilis em jovens conscritos brasileiros: uma investigação descritiva [dissertação]. Brasília (DF): Universidade de Brasília;2018. Disponível em: https:// repositorio.unb.br/bitstream/10482/33017/1/2018_ FranciscaLidianeSampaioFreitas.pdf

19. Ferreira-Júnior 0, Guimarães MDC, Damacena GN, Almeida WS, Souza-Júnior PRB, Szwarcwald CL.Prevalence estimates of HIV, syphilis, hepatitis B and $\mathrm{C}$ among female sex workers (FSW) in Brazil, 2016.Medicine (Baltimore) [Internet]. 2018 May [citado 2020 jun 09];97(1Suppl):S3-8. Disponível em: https://doi.org/10.1097/md.0000000000009218

20. Ministério da Saúde (BR). Secretaria de Vigilância em Saúde. Departamento de Doenças de Condições Crônicas e Infecções Sexualmente Transmissíveis. Sífilis, 2019. BolelEpidemiol [Internet]. 2019 out [citado 2020 ago 31];5(1). Disponível em: http://www.aids.gov.br/ pt-br/pub/2019/boletim-epidemiologico-sifilis-2019

21. PanAmerican Health Organization - PAHO. New generations free of HIV, syphilis, hepatitis $b$, and chagas disease: EMTCT plus in the Americas, 2018 [Internet]. Washington, D.C.: PanAmerican Health Organization; 2019 [citaed 2020 Jun 25]. 42p. Available from: https:/www.paho.org/en/documents/ new-generations-free-hiv-syphilis-hepatitis-b-and-chagas-disease-americas-emtct-plus-2018

22. Soeiro CMO, Miranda AE, Saraceni V, Santos MC, Talhari S, Ferreira LCL. Syphilis in pregnancy and congenital syphilis in Amazonas State, Brazil: an evaluation using database linkage. CadSaúde Pública [Internet]. 2014 Apr [cited 2020 Jul 30];30(4):715-23. Available from: https://doi.org/10.1590/0102-311X00156312
23. Lafeta KRG, Martelli Júnior H, Silveira MF, Paranaíba LMR. Sífilis materna e congênita, subnotificação e difícil controle. Rev Bras Epidemiol [Internet]. 2016 mar [citado 2020 jul 30];19(1):63-74. Disponível em: https://doi.org/10.1590/1980-5497201600010006

24. Ministério da Saúde (BR). Secretaria de Vigilância em Saúde. Departamento de Vigilância, Prevenção e Controle das Infecções Sexualmente Transmissíveis do HIV/Aids e Hepatites Virais. Nota Informativa $n^{0}$ 2 , de 19 de setembro de 2017. Altera os critérios de definições de casos para notificação de sífilis adquirida, sífilis em gestantes e sífilis congênita [Internet]. Brasília: Ministério da Saúde; 2017 [citado 2020 jul 31]. (SEI/MS n ${ }^{0}$ 0882971). Disponível em: http:// portalsinan.saude.gov.br/images/documentos/ Agravos/Sifilis-Ges/Nota_Informativa_Sifilis.pdf

25. Ministério da Saúde (BR). Secretaria de Vigilância em Saúde. Programa Nacional de DST e AIDS. Curso básico de vigilância epidemiológica em HIV e aids: caderno do aluno. Brasília: Ministério da Saúde; 2005.

26. Waldman EA. Vigilância em saúde pública, volume 7 [Internet]. São Paulo: Faculdade de Saúde Pública da Universidade de São Paulo; 1998 [citado 2020 ago 31]. (Série Saúde \& Cidadania). 253 p. Disponível em:http:// ead.saude.riopreto.sp.gov.br/pluginfile.php/303/course/ summary/Saude\%20\%20Cidadania\%20Volume07.pdf

27. Tayra Â, Matida LH, Saraceni V, Paz LC, Ramos Júnior AN. Duas décadas de vigilância epidemiológica da sífilis congênita no Brasil: a propósito das definições de caso. DST - J Bras Doenças Sex Transm [Internet]. 2007 nov [citado 2020 ago 31];19(3-4):111-9. Disponível em: http://www3.crt.saude.sp.gov.br/tvhivsifilis/ artigos_manuais_textos/historia_ve_sifilis.pdf

28. Lago EG, Vaccari A, Fiori RM. Clinical features and follow-up of congenital syphilis. Sex Transm Dis [Internet]. 2013 Feb [cited 2020 Aug 31];40(2):85-94. Available from: https:// doi.org/10.1097/olq.0b013e31827bd688

29. Cavalcante ANM, Araújo MAL, Nobre MA, Almeida RLF. Fatores associados ao seguimento não adequado de crianças com sífilis congênita. Rev Saúde Pública[Internet]. 2019 out [citado 2020 jul 31];53:95. Disponível em: https:// doi.org/10.11606/s1518-8787.2019053001284

30. Ministério da Saúde (BR). Secretaria de Vigilância em Saúde. Coordenação-Geral de Vigilância das Infecções Sexualmente Transmissíveis. Vigilância epidemiológica das infecções sexualmente 
transmissíveis no Brasil. Bol Epidemiol [Internet]. 2020 fev [citado 2020 jun 9];51(8):11-5. Disponível em: https://www.saude.gov.br/images/pdf/2020/ fevereiro/21/Boletim-epidemiologico-SVS-08.pdf

31. Bazzo ML, Golfetto L, Gaspar PC, Pires AF, Ramos MC, Franchini M, et al. First nationwide antimicrobial susceptibility surveillance for Neisseria gonorrhoeae in Brazil, 2015-16. J Antimicrob Chemother [Internet]. 2018 Jul [cited 2020 Aug 31];73(7):1854-61. Available from: https://doi.org/10.1093/jac/dky090

32. Benzaken AS, Pereira GFM, Cunha ARC, Souza FMA, Saraceni V. Adequacy of prenatal care, diagnosis and treatment of syphilis in pregnancy: a study with open data from Brazilian state capitals. Cad Saúde Pública [Internet]. 2020 Dec [cited 2020 Aug 01]; 36(1):e00057219. Available from: https://doi.org/10.1590/0102-311x00057219

33. Nonato SM, Melo APS, Guimarães MDC. Sífilis na gestação e fatores associados à sífilis congênita em Belo Horizonte-MG, 2010-2013. Epidemiol Serv Saúde [Internet]. 2015 out-dez [citado 2020

\section{Abstract}

This article summarizes the chapter on epidemiological surveillance of sexually transmitted infections (STI) that forms part of the Clinical Protocol and Treatment Guidelines (PDCT) for Comprehensive Care for People with STI 2020), published by the Health Surveillance Secretariat of the Ministry of Health of Brazil. It presents some reflections on the new case definitions for surveillance of acquired syphilis, syphilis in pregnant women and congenital syphilis. The PCDT-IST 2020 was developed based on scientific evidence and validated in discussions with specialists. Epidemiological and clinical aspects are addressed, as well as guidelines for bealth service managers regarding programmatic and operational management of these diseases, guidelines for health professionals on screening, diagnosing and treating people with STIs and their sex partners, in addition to strategies for surveillance, prevention and control actions.

Keywords: Public Health Surveillance; Health Information Systems; Syphilis; Syphilis, Congenital; Disease Notification; Epidemiological Monitoring. ago 31];24(4):681-94. Disponível em: https:// doi.org/10.5123/S1679-49742015000400010

34. Sanine PR, Castanheira ERL, Nunes LO, Andrade MC, Nasser MA, Nemes MIB. Sífilis congênita: avaliação em serviços de Atenção primária do estado de São Paulo, Brasil. BIS Bol Inst Saúde (impr) [Internet]. $2016 \mathrm{dez}$ [citado 2020 ago 31];17(2):128-37. Disponível em: http://docs.bvsalud.org/biblioref/2019/10/1021661/ bis-v17n2-saude-e-direitos-sexuais-128-137.pdf

35. Domingues CSB, Pinto VM. Congenital syphilis in the 21st century: how to overcome the challenges? DST - J Bras Doenças Sex Transm [internet]. 2019 [cited 2020 Aug 31];31(3):77-8. Available from: https://doi.org/10.5327/DST-2177-8264-201931301

36. Saraceni V, Pereira GFM, Silveira MF, Araujo MAL, Miranda AE. Vigilância epidemiológica da transmissão vertical da sífilis: dados de seis unidades federativas no Brasil. Rev Panam Salud Pública [Internet]. 2017 abr [citado 2020 ago 01]; 41:e44. Disponível em: https://dx.doi.org/10.26633\%2FRPSP.2017.44

\section{Resumen}

El artículo resume el capítulo sobre vigilancia epidemiológica de las infecciones de transmisión sexual (ITS) que comprende el Protocolo Clínico y Directrices Terapéuticas (PDCT) para Atención Integral a las Personas con IST 2020, publicado por la Secretaría de Vigilancia Sanitaria del Ministerio de Salud de Brasil y presenta algunas reflexiones sobre las nuevas definiciones de casos de la vigilancia de sífilis adquirida, sífilis en mujeres embarazadas y sífilis congénita. El PCDT-IST 2020 fue elaborado en base a la evidencia científica y validado en discusiones con especialistas. En este artículo, se presentan aspectos epidemiológicos y clínicos, así como pautas para los gerentes en el manejo programático y operativo de estas enfermedades, a los profesionales de la salud en la detección, diagnóstico y tratamiento de personas con ITS y sus parejas sexuales, además de estrategias para acciones de vigilancia, prevención y control.

Palabras clave: Vigilancia en Salud Pública; Sistemas de Información en Salud; Sífilis; Sífilis Congénita; Notificación de Enfermedades; Monitoreo Epidemiológico.

Recebido em 09/07/2020

Aprovado em 14/08/2020 